ganglion." Treatment consists of synovectomy and antituberculous chemotherapy. Acute non-suppurative arthritis occurs only rarely in the hand. Gout may go unsuspected for some time.

If antibiotics are administered inappropriately the course of the infection is frequently modified to the patient's detriment. Chronic infection may result, with swelling, induration, and stiffness of the digit or hand. This may be difficult to resolve and may, indeed, result in persistent functional disability. Changes in the articular cartilage of infected joints progress rapidly and move toward secondary osteoarthrosis. Adhesions in the flexor tendon apparatus may produce severe limitation of the excursion of the flexor tendons and require tenolysis.

The prescription of antibiotics as the sole treatment of a hand infection must therefore be undertaken with circumspection. The penalty for errors is great. The result of a hand infection, or a hand injury, is almost always related to the quality of the primary treatment. Primary treatment cannot be instituted correctly unless the diagnosis is also correct. When there is doubt, therefore, the patient should be referred to a hand surgeon immediately.

NeIL Watson

Consultant Hand Surgeon,

Milton Keynes Hospital,

Milton Keynes MK6 5LD

1 Bailey D. The infected hand. London: H K Lewis, 1963.

Carter PR. Common hand injuries and infections. A practical approach to early treatment. Philadelphia: W B Saunders, 1983.

3 Clarkson P, Pelly A. The general and plastic surgery of the hand. Oxford: Blackwell Scientific Publications, 1962

4 Flynn JE. Hand surgery. Baltimore: Williams and Wilkins, 1975.

5 Lamb DW, Kuczynski K. The practice of hand surgery. Oxford: Blackwell Scientific Publications, 1981

6 Lister G. The hand: diagnosis and indications. Edinburgh: Churchill Livingstone, 1977.

7 Milford L. The hand in Campbell's operative orthopaedics. St Louis: C V Mosby, 1981

8 Sneddon J. The care of hand infections. London: Edward Arnold, 1970.

9 Watson N. Hand injuries and infections. London: Gower (in press

10 Watson N. Practical management of musculoskeletal emergencies. Oxford: Blackwell Scientific Publications (in press).

\title{
New hepatitis B vaccines
}

\author{
ARIE J ZUCKERMAN
}

Viral hepatitis is a major public health problem in all parts of the world. The infection may be caused by any of half a dozen viruses: hepatitis $\mathrm{A}$, hepatitis $\mathrm{B}$, hepatitis $\mathrm{D}$ (the delta agent, a defective virus), epidemic non-A hepatitis, and by at least two non- $A$, non- $B$ viruses.

Hepatitis B (fig 1) affects every part of medical practice and its importance cannot be exaggerated. Infection may progress to chronic liver disease, including chronic persistent and chronic active hepatitis, cirrhosis, and hepatocellular carcinoma, one of the most common malignant tumours. Persistent infection is common, particularly if acquired early in life, and at a conservative estimate there are 200 million carriers of hepatitis B world wide.' Immunisation against hepatitis B is, therefore, required for groups at high risk of infection, as assessed by epidemiological patterns, socioeconomic factors, cultural and sexual practices, and the environment. ${ }^{2}$

The high rates of infection and perinatal transmission of hepatitis B in some regions dictate the urgency of protective immunisation of susceptible women of childbearing age and of infants-and particularly of infants born to carrier mothers-as the only practical way of interrupting transmission of the infection. ${ }^{3}$ Immunisation must also be considered for people living in certain tropical and non-tropical areas where the prevalence of hepatitis B infection is high, where $10-20 \%$ or more of the population may be carriers, and where primary liver cancer is common.

The development, safety, and efficacy of hepatitis B vaccine consisting of the excess surface antigen protein coat of the virus purified from the plasma of asymptomatic carriers (fig 2) have been reviewed recently. ${ }^{2}$ This article describes the rapid advances which are being made with polypeptide vaccines, hepatitis $B$ vaccines produced by recombinant DNA technology, recombinant live hepatitis B vaccines, and chemically synthesised vaccines.

\section{Hepatitis B polypeptide vaccines}

There are two main polypeptides of purified hepatitis B surface antigen, one with a molecular weight of 22000 to 25000 or 26000 (variations exist among the results of analyses of purified antigen from different sources) and the other, its glycosylated form, with a molecular weight of 28000-30000. These have been designated p25 and gp30. The polypeptide vaccines contain both p25 and gp 30 and have been prepared and tested for safety, immunogenicity, and protective efficacy in susceptible chimpanzees. ${ }^{45}$ When compared with the plasma vaccine these polypeptide vaccines are better defined chemically and have an added margin of 


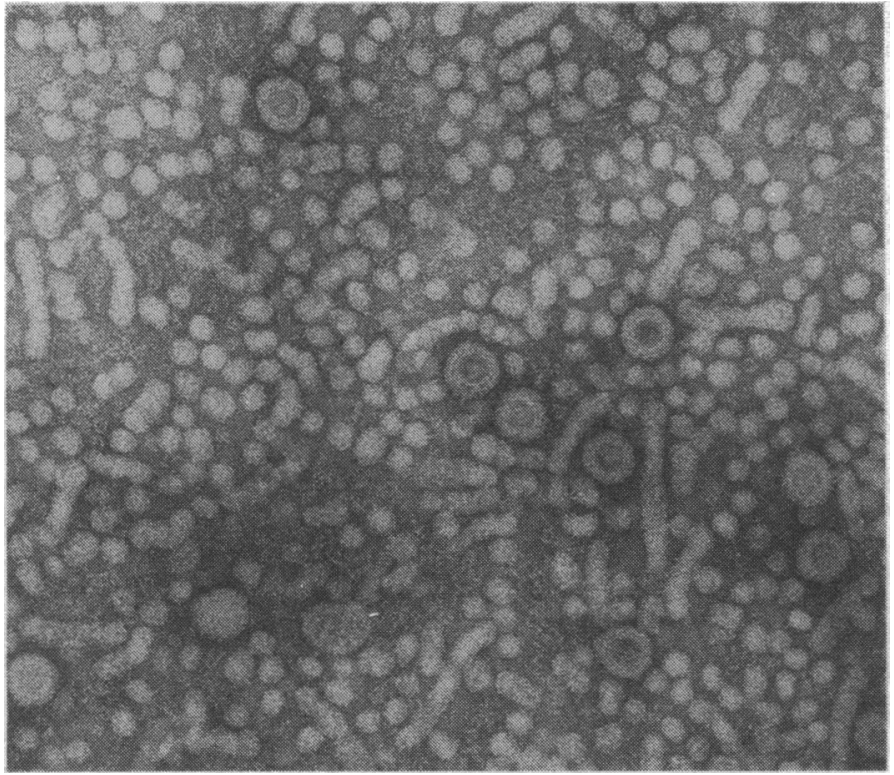

FIG 1-Electronmicrograph showing complex morphology of hepatitis B virus in serum. (1) Large double shelled complete virus particles about $42 \mathrm{~nm}$ diameter. (2) Small spherical particles of hepatitis B surface antigen about $22 \mathrm{~nm}$ diameter. (3) Tubular forms of surface antigen $\times 125000$ (original magnification).

safety since they would be even less likely to contain contaminating virus or host proteins.

Several studies have shown that individual polypeptides of the surface antigen are immunogenic, but, more particularly, that purified polypeptides with molecular weights of 25000 and 30000 are effective antigens. ${ }^{67}$ The main difficulty in obtaining sufficient quantities of the peptides in pure form was overcome by developments at the London School of Hygiene and Tropical Medicine. The purification of viral coat subunits in large quantities presents considerable problems with viruses possessing a lipoprotein envelope, where the immunogenic components are integral membrane proteins, highly hydrophobic, insoluble in aqueous media, and requiring drastic treatment with detergents. The extraction of the antigenic polypeptides with the non-ionic detergent Triton X-100 resolved one of the problems.

Polypeptides in monomeric solution in high concentrations of detergent are not, however, a suitable form of vaccine; so a method of detergent removal was developed

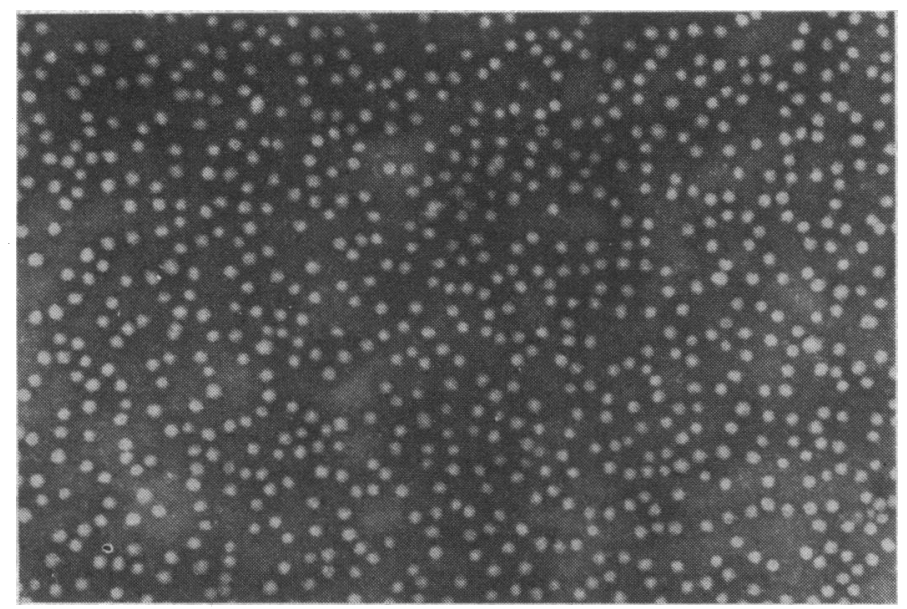

FIG 2-Hepatitis B small particle ( $22 \mathrm{~nm}$ ) vaccine after purification from pooled plasma and inactivation. $\times 100000$ (original magnification). which allowed membrane polypeptides to reassociate into water soluble protein micelles. Protein micelles are aggregates of polypeptides arranged so that the hydrophobic regions are sequestered in the interior of the particles with the hydrophilic residue on the surface, and the resulting particulate forms are water soluble. Hepatitis B polypeptide micelle vaccine (fig 3) was prepared in this way, and comparison of the immunogenicity of the micelles with the plasma vaccine

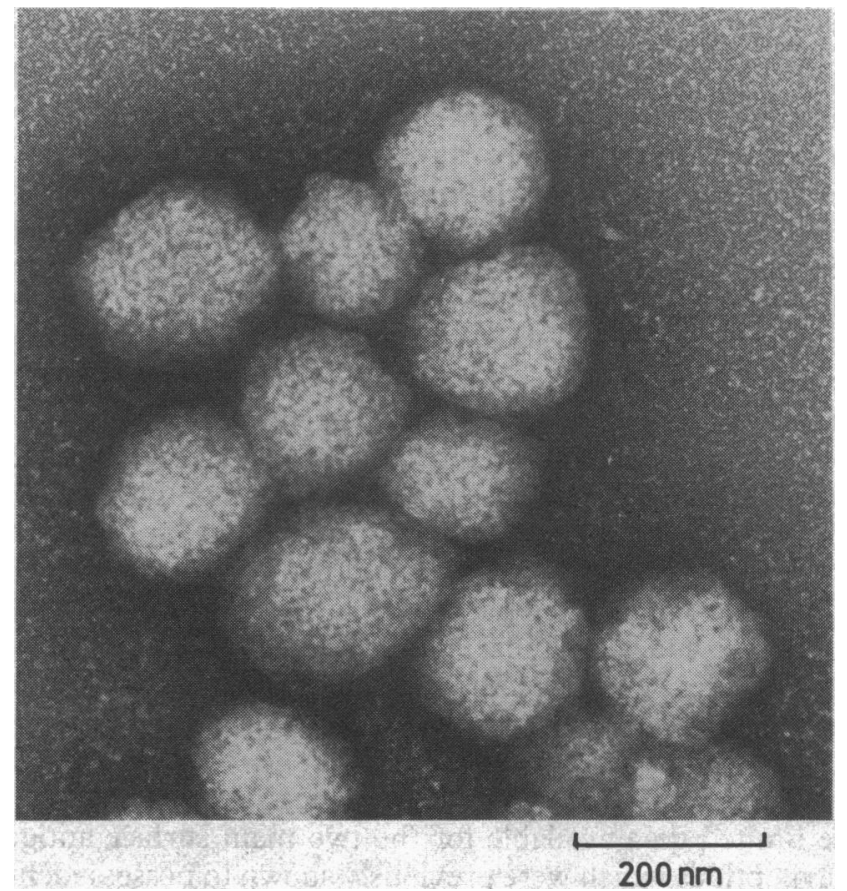

FIG 3-Hepatitis B polypeptide vaccine in micelle form. Large surface area of micelles together with altered distribution of antigenic sites and absence of host derived proteins may account for enhanced antibody response.

in a mouse potency assay showed that the micelles elicited a more vigorous protective surface antibody response than the intact particles at all dose concentrations tested. ${ }^{67}$ Safety tests and protective efficacy studies in primates have been completed, ${ }^{5}$ and clinical trials of the micelle vaccine are in progress. Polypeptide micelles have also been prepared from antigen expressed by recombinant DNA technology in yeast and in mammalian cells, as discussed below.

\section{Recombinant DNA techniques}

Advances in molecular genetics and nucleic acid chemistry have permitted the identification and analysis of genes coding for biologically active substances, transfer within and between organisms, and expression under controlled conditions. A gene which codes for a specific product may be isolated and propagated by inserting naturally occurring or synthetic DNA molecules into a suitable vector, which is then introduced into a host; individual clones carrying the required gene are then selected. A key step in the process is the insertion of the gene into the vector with the aid of highly specific restriction endonucleases (enzymes which cleave the vector DNA at predetermined sites) and ligases (which join the gene insert to the vector).

A gene is characterised by a specific nucleotide sequence in one strand of the double stranded DNA molecule. When the 
strands are separated each forms a template for the synthesis of a complementary copy, thereby providing a mechanism for accurate reproduction of genes with conservation of the linear sequence of the four nucleotides. The process of decoding this information and the synthesis of the gene product occurs by transcription of the DNA coding strand in the form of a messenger RNA (mRNA), followed by translation of the information carried by the mRNA into an amino acid sequence.

Recombinant DNA techniques have been used for expressing hepatitis B surface antigen in prokaryotic cells (Escherichia coli and Bacillus subtilis) and in eukaryotic cells (such as mutant mouse LM cells, HeLa cells, COS cells, and, particularly important, in yeast cells, Saccharomyces cerevisiae). These studies have established the nucleotide sequence of hepatitis B DNA, the organisation of the viral genome, and the prediction of amino acid sequences of the major gene products (reviewed by Howard and Zuckerman ${ }^{10}$ ). The genome contains four main polypeptide "reading frames," which theoretically may be available for transcription in a naturally infected hepatocyte from the long DNA strand of the double stranded viral genome. The "S" gene codes for a protein of molecular weight 25000 which resembles closely in predicted sequence the non-glycosylated major hepatitis B surface antigen polypeptide (p25) identified by electrophoretic analysis of $22 \mathrm{~nm}$ particles purified from plasma of infected patients. Another reading frame, the "C" gene, codes for the 21000 molecular weight viral core polypeptide. A third reading frame overlaps the " $S$ " gene, and the fourth partially overlaps the " $C$ " gene.

The amino acid sequence of the " $S$ " gene product may be predicted from the nucleotide sequence and compared with the limited data available for the two main surface antigen polypeptides which were previously shown to possess identical amino and carboxy terminal sequences. ${ }^{11}$ The full 226 amino acid sequence contains a relatively high content of aromatic and hydrophobic regions. There are, however, minor amino acid changes between different and even within a similar subtype of the virus, but these variations are beyond the scope of this review. ${ }^{10}$

Expression of cloned genes in yeast ( $S$ cerevisiae) was an important advance which had considerable advantages for expression of hepatitis B surface antigen. These include the presence of Golgi like secretory apparatus for protein maturation and much higher yields of the requisite gene product owing to the availability of a eukaryote codon usage system for RNA recognition during translation. This was first achieved by using an autonomously replicating plasmid containing the " $S$ " gene of hepatitis B virus linked to a yeast alcohol dehydrogenase I promoter. ${ }^{12}$ The surface antigen synthesised in yeast is particulate but cell associated. After disruption of the cells the particles measure $17-20 \mathrm{~nm}$ diameter, slightly smaller than the natural particles found in the plasma of infected patients. Chemical analysis shows that only the non-glycosylated polypeptide (p25) is present, but the particles are fully antigenic and immunogenic, and when formulated as a vaccine they protect immunised susceptible chimpanzees against challenge with infectious virus. ${ }^{13}$ This and other recombinant yeast hepatitis B vaccines are being evaluated by clinical trials. ${ }^{14}$ Unpublished results indicate that this vaccine is safe, antigenic, and free from side effects (apart from minor local reactions). The immunogenicity with a dose of $10 \mu \mathrm{g} / \mathrm{ml}$ as compared with $20 \mu \mathrm{g} / \mathrm{ml}$ of the plasma vaccine, is similar. The antigen expressed in yeast has been prepared in a micellar form (by C R Howard, P Young, A J Zuckerman, et al (to be published) and by Burnette $e t a l^{9}$ ).
The yeast derived polypeptide micelles were found to be considerably more antigenic with enhanced immunogenic potency.

\section{Construction of live hepatitis B vaccines with genetically engineered vaccinia virus}

Potential live vaccines by using recombinant vaccinia viruses have been constructed for hepatitis $\mathrm{B},{ }^{15}$ and also for herpes simplex, ${ }^{16}$ rabies, ${ }^{17}$ and others. Foreign viral DNA is introduced into the vaccinia DNA by construction of chimeric genes. This is accomplished by homologous recombination in cells, since the large size of the genome of vaccinia virus (185000 base pairs) precludes gene insertion in vitro. A chimeric gene consisting of vaccinia virus promoter sequences ligated to the coding sequence for the desired foreign protein is flanked by vaccinia virus DNA in a plasmid vector. Plasmids were constructed with restriction enzyme sites next to the vaccinia thymidine kinase promoter or another promoter engineered in the thymidine kinase gene. The thymidine kinase locus has the advantage that recombinants resulting from transfection of vaccinia virus infected cells with the plasmid may be selected on the basis of the altered phenotype.

The recloned vaccinia virus containing hepatitis B surface antigen coding sequences were used to vaccinate rabbits with the production of typical vaccinia lesions and high titres of hepatitis B surface antibody. ${ }^{15}$ The results of preliminary studies in chimpanzees were encouraging, showing that the vaccinated chimpanzees had a secondary antibody response when challenged with live hepatitis B virus of a heterologous subtype with a mild inapparent infection characterised by seroconversion to surface antibody and hepatitis B anticore. ${ }^{18}$

At present, however, there is no generally accepted laboratory marker of attenuation or of virulence of vaccinia virus for man, not only in the host directly inoculated with the material but also after several passages in the same species. Alterations in the genome of vaccinia virus which are concomitant with the selection of recombinants may alter the virulence of the virus. Interruption of the thymidine kinase gene of the virus by insertion of foreign DNA was noted to reduce its virulence for mice. Nevertheless, changes in host range or tissue tropism of vaccinia viruses may occur as a result of their genetic modification, and these might be caused by changes in the virus envelope as a result of the incorporation of gene products of the foreign viral genes inserted into the vaccinia virus.

The potential advantages of vaccinia as a live virus vaccine include low cost, ease of administration by multiple pressure or by the scratch technique, vaccine stability, long shelf life, and the use of polyvalent antigens. The known adverse reactions with vaccinia virus vaccines are well documented, ${ }^{19}$ and their incidence and severity must be weighed against the adverse reactions associated with existing vaccines which a new recombinant vaccine might replace.

\section{Chemically synthesised hepatitis $B$ vaccines}

The ultimate goal of vaccine development is production of chemically uniform and safe viral immunogens to replace the many current vaccines which contain large quantities of irrelevant microbial antigenic determinants, proteins, and other material additional to the essential immunogen required for the induction of a protective antibody. Synthetic 
polypeptide vaccines offer many advantages in this respect. Certainly the preparation of antibodies against viral proteins using fragments of chemically synthesised peptides mimicking viral amino acid sequences is now a possible alternative approach in immunoprophylaxis. The feasibility of synthetic vaccines was first shown in studies with tobacco mosaic virus after the identification of an antigenic determinant and its amino acid sequences responsible for the immunogenic activity of the virus (reviewed by Arnon ${ }^{20}$ ). Such amino acid moieties may be synthesised, and, when coupled to a carrier protein, they induce high titres of neutralising antibody in animals.

More recent studies have included the induction of an immune response to an intact strain of human influenza $A$ virus using a synthetic peptide analogous to a sequence of the haemagglutinin of $\mathrm{H} 3 \mathrm{~N} 2$ influenza $A$ virus. ${ }^{21}$ The peptide was covalently linked to several macromolecular carriers, and the conjugate with tetanus toxoid was used for the immunisation of rabbits and mice with the production of specific antibodies. These antibodies were protective against infection with a relatively low viral challenge in mice with the $\mathrm{A} / \mathrm{Texas} /$ 77 mouse adapted influenza virus. The results show the potential of synthetic material for eliciting antiviral immunity against an important and common human pathogen.

Veterinary vaccines have also been synthesised. For example, synthesised peptides corresponding to two regions of the major VPI polypeptide of foot and mouth disease virus coupled to a protein carrier produced high titres of specific neutralising antibody in several animal species including cattle, and immunised guinea pigs were fully protected against virulent virus after receiving only a single dose of one peptide. ${ }^{22}$

Information on the use of synthetic peptides is also available from antibodies induced against bacterial toxin. Diphtheria toxin is a single polypeptide chain (molecular weight 62000 ) with two disulphide bridges. The loop of 14 amino acids subtended by the disulphide bridge near the $\mathrm{NH}_{2}$ terminus is probably implicated in the toxicity and immunological specificity of the molecule. A synthetic tetradecapeptide mimicking a portion of this active site linked covalently to two different carriers elicited antibodies in guinea pigs which bound specifically with the toxin and neutralised its dermonecrotic and lethal effects. ${ }^{23}$ Another example is the induction of type specific protective immunity using a synthetic peptide of Streptococcus pyogenes M protein which contained only 12 amino acid residues. ${ }^{24}$ The immunogenicity of such small peptides indicates a way to the development of safe vaccines against the streptococcal infections which cause rheumatic fever and rheumatic heart disease. In more general terms the efficacy of the very small peptides would permit the disposal of a large portion of the protein molecule. This should also reduce the chances of eliciting immunological cross reactions against host tissues.

Similar approaches to the development of chemically synthesised hepatitis B vaccines were suggested a few years ago by several investigators, ${ }^{25-27}$ and current progress suggests that such synthetic peptide vaccines are now within reach. ${ }^{28}$

The strategy for producing synthetic material adapted by laboratories consists of four stages. Firstly, the antigenic portions of the " $\mathrm{S}$ " gene product are predicted directly by analysis of its amino acid sequence. Computer analysis is used to locate the points of greatest hydrophilicity in the polypeptide chain. Next comes the synthesis of selected sequences, which generally use the classical solid phase method of Erickson and Merrifield. Finally, the antigenic and immunogenic properties of synthesised fragments are assessed, either directly or after coupling to a carrier protein.

Several laboratories have reported independently the successful mimicking of determinants of hepatitis B surface antigen using chemically synthesised peptides. In one laboratory the sequence between residues 138 and 149 of the surface antigen which contained a putative dominant epitope in positions 141-146 was selected. ${ }^{29}$ The four cysteinyl residues were replaced by $\alpha$ aminobutyric acid to prevent polymerisation and other side reactions common to sulphydryl containing peptides, and glycine was added to the $\mathrm{COOH}$ terminus to aid radioimmunoassay directly on the solid phase used for synthesis. The prediction that the synthetic peptide contained a main epitope of hepatitis B surface antigen was confirmed, this sequence of amino acids eliciting antibodies directed to group specific determinant $a$ and the subdeterminant $d$, but not the epitope of subdeterminant $y$ or that of albumin added to a control. When the peptide was attached to aldehyde stabilised human erythrocytes and injected into mice it induced the formation of hepatitis B surface antibody with and without the use of Freund's complete adjuvant. ${ }^{30} \mathrm{~A}$ synthetic oligopeptide of 13 amino acids in the sequence between 136 and 147 had been reported to represent a partial analogue of the $a$ determinant of the surface antigen. ${ }^{31}$

The results of a somewhat different approach using two predicted hydrophilic regions of the hepatitis B surface antigen molecule were described from another laboratory. ${ }^{32}$ Two cyclic peptides containing disulphide bonds in the region between the amino acid sequences 117-137 and 112137 were incorporated into several adjuvants including Freund's complete adjuvant, alum, and multilamellar liposomes with and without muramyl dipeptide. Groups of mice were immunised intraperitoneally with each of the preparations. Hepatitis B surface antibody was induced seven to 14 days after inoculation in roughly half of the mice in each group and in four or five out of six mice when the immunising preparation of the 117-137 peptide was emulsified in Freund's complete adjuvant. On day 21 , however, the peak concentrations of antibody decreased in most groups of mice. The antibody response was elicited in mice after a single injection without covalent linkage to a carrier protein, and the choice of adjuvant did not affect the primary antibody response. Furthermore, the hepatitis B surface antibody response in mice inoculated with p25 solubilised with sodium dodecyl sulphate was similar to that observed with the synthetic peptides.

Several other synthetic peptides were examined in another laboratory but avoiding the region 110-140 because of published differences in nucleotide sequences in this region. The 13 peptides included sequences at the amino and carboxyl termini, peptides corresponding to hydrophilic domains of the protein, and peptides likely to contain exposed folds in the peptide chain indicated by proline containing junctions between hydrophilic and hydrophobic regions. ${ }^{33}$

Seven out of the 13 free or protein carrier linked synthetic peptides elicited an antipeptide response in rabbits. Where used the carrier protein was keyhole limpet haemocyanin in complete and subsequently incomplete Freund's adjuvant. Antisera against four out of the six soluble peptides, ranging from 10 to 34 amino acid residues, reacted with the native antigen and also precipitated the 25000 (p25) and 30000 (gp30) molecular weight polypeptides of hepatitis B surface antigen. Possibly short peptides may be immunogenic when free of constraints imposed by neighbouring amino acids which are manifested by denaturation of the native molecule; in theory the short peptides are free to assume all possible 
configurations at any one time, one of which resembles the native molecule and thus stimulates antibody production.

Recently a peptide with the sequence $110-137$ was reported to stimulate a transitory antibody response to one of the surface antigen determinants $y$ in inoculated chimpanzees. Repeated immunisation of the chimpanzees failed to induce a stable surface antibody response. Nevertheless, intravenous challenge with hepatitis B virus with the ayw determinants late in the course of immunisation showed protection against infection in one chimpanzee, an attenuated infection without disease in another, and typical acute hepatitis in the third. ${ }^{34}$

Thus chemical synthesis of small peptides representing specific regions of hepatitis B surface antigen is now feasible. Antisera to these peptides cross react with the native surface antigen particles. A different approach was used at the London School of Hygiene and Tropical Medicine. Antibodies to a chemically synthesised nonapeptide representing amino acids $139-147$ of the 226 amino acids molecule were shown to bind antibodies present in hepatitis B immunoglobulin and in the sera of patients recovered from acute hepatitis B and monoclonal anti- $a$ and antibodies in the serum of people immunised with the plasma derived hepatitis $B$ vaccine licensed in Britain. ${ }^{12}$ The affinity and level of hepatitis B surface antibody were determined with three different antigens, synthetic linear and cyclical amino acid sequences 139-147 and the p25-gp30 complex of the native surface antigen prepared by solubilisation. ${ }^{6}$ Antibody values, expressed as total antibody combining sites in fixed volumes of immune sera, increased throughout the course of immunisation and correlated with the development of surface antibody as measured by radioimmunoassay. The values of the total antibody combining sites were similar for both forms of the synthetic peptide, though higher affinity values were found with the cyclical structure, which shows the importance of protein conformation in antibody responses to hepatitis B surface antigen. ${ }^{35} 36$
Antibody affinity for the three antigens increased progressively throughout the immunisation schedule, but the pattern of affinity maturation was better for the native $\mathrm{p} 25$ gp30 complex and the synthetic cyclical peptide than the linear peptide. Furthermore, the affinity of the antibody response in healthy people to three doses of the vaccine was similar to the affinity of the antibody response to natural infection, in that after the third (booster) dose of vaccine all subjects had affinity values which fell within, or were greater than, the range of affinities of the patients recovered from acute hepatitis B. ${ }^{35} 36$

Synthetic peptides may therefore be employed in due course as vaccines, though mixtures of more than one of the peptides may be required. Of the many questions which remain to be answered, the critical issues are whether antibodies induced by synthetic immunogens will be protective and whether protective immunity will persist. Some of the carrier proteins and some of the adjuvants which had been linked to the synthetic molecules cannot be used in man, and acceptable and safe material needs to be found for covalent linkage, or, alternatively, sequences which do not require linkage may be synthesised.

We are now entering the era of antigen and antibody engineering, and the prospect of multivalent synthetic vaccines against a variety of microbial agents is within reach.

The hepatitis B vaccine development programme at the London School of Hygiene and Tropical Medicine is generously supported by the Department of Health and Social Security, the Wellcome Trust, the British Technology Group, and the Commission of the European Economic Community.

ARIE J ZuCKerman

Professor of Microbiology and Director of the WHO

Collaborating Centre for Reference and Research on Viral Hepatitis, London School of Hygiene and Tropical Medicine, London WC1E 7HT
1 World Health Organisation. Prevention of liver cancer. WHO Tech Rep Ser 1983; No 691.

2 Zuckerman AJ. Who should be immunised against hepatitis B? Br Med f 1984;289:1243-4

3 Zuckerman AJ. Perinatal transmission of hepatitis B. Arch Dis Child 1984;59:1007-9.

4 Dreesman GR, Hollinger FB, Sanchez Y, Oefinger P, Melnick JL. Immunisation of chimpanzees with hepatitis B virus-derived polypeptides. Infect Immun 1982;32:62-7.

5 Tabor $\mathrm{E}$, Howard CR, Skelly J, et al. Immunogenicity in chimpanzees of experimental hepatitis B vaccines made from intact hepatitis B virus, purified polypeptides or polypeptides in micelles. f Med Virol 1982;10:65-74.

6 Skelly J, Howard CR, Zuckerman AJ. Hepatitis B polypeptide vaccine preparation in micelle form. Nature 1981;290:51-4

7 Sanchez Y, lonescu-Matiu I, Melnick JL, Dreesman GR. Comparative studies of the immunogenic activity of hepatitis B surface antigen $(\mathrm{HBsAg})$ and $\mathrm{HBsAg}$ polypeptides. $\mathcal{J}$ Med Viro 1983;11:115-24

8 Howard CR, Young PR, Tsiquaye KN, Zuckerman AJ. Developments of hepatitis B polypeptide micelle vaccines. In: Channock RM, Lerner RA, eds. Modern approaches to vaccines. New York: Cold Spring Harbor Laboratory, 1984:385-91

9 Burnette WN, Samal B, Browne JK, Fenton D, Bitter GA. Production of hepatitis B recombinan vaccines. In: Channock RM, Lerner RA, eds. Modern approaches to vaccines. New York: Cold Spring Harbor Laboratory, 1984:245-50.

i0 Howard CR, Zuckerman AJ. New viral hepatitis vaccines. In: Kurstak E, Marusyk RG, eds. Control of virus diseases. New York: Marcel Dekker, 1984:33-55.

11 Peterson DL. Isolation and characterisation of the major protein and glycoprotein of hepatitis $\mathrm{B}$ surface antigen. I Biol Chem 1981;256:6975-83.

12 Valenzuela P, Medina A, Rutter WJ. Synthesis and assembly of hepatitis B virus surface antigen particles in yeast. Nature 1982;298:347-50.

13 McAleer WJ, Buynak EB, Maigetter RZ, Wampler DE, Miller WJ, Hilleman MR. Human hepatitis B vaccine from recombinant yeast. Nature 1984;307:178-80

14 Jilg W, Lobeer B, Schmidt M, Wilkse B, Zoulek G, Deinhardt F. Clinical evaluation of a recombinant hepatitis $B$ vaccine. Lancet 1984 ;ii: $1174-5$.

15 Smith GL, Mackett $M$, Moss B. Infectious vaccinia virus recombinants that express hepatitis $B$ virus surface antigen. Nature 1983;302:490-5.

16 Paoletti E, Lipinskas BR, Samsonoff C, Mercer S, Panicali D. Construction of live vaccines using genetically engineered poxviruses: biological activity of vaccinia virus recombinants expressing the hepatitis B surface antigen and the herpes simplex virus glycoprotein D. Proc Natl Acad Sc USA 1984;81:193-7.

17 Kieny MP, Lathe $\mathrm{R}$, Drillien $\mathrm{R}$, et al. Expression of rabies virus glycoprotein from a recombinant vaccinia virus. Nature 1984;312:163-6.

18 Moss B, Smith GL, Gerin JL, Purcell RH. Live recombinant vaccinia virus protects chimpanzees against hepatitis B. Nature 1984;311:67-9.
19 Wilson GS. The hazards of immunization. London: Athlone Press, 1967:157-78.

20 Arnon R. Identification of antigenic determinants and their application in synthetic vaccines. In: Bell R, Torrigiani G, eds. New Approaches to vaccine development. Basle: Schwabe, 1984:44-65.

21 Muller GM, Shapira M, Arnon R. Anti-influenza response achieved by immunisation with a synthetic conjugate. Proc Natl Acad Sci USA 1982;79:569-73.

22 Bittle JL, Houghten RA, Alexander H, Shinnick TM, Sutcliffe JG, Lerner RA. Protection against foot-and-mouth disease by immunisation with a chemically synthesized peptide predicted from the viral nucleotide sequence. Nature 1982;298:30-3.

23 Audibert F, Jolivet $M$, Chedid L, et al. Active antitoxic immunization by a diphtheria toxin synthetic oligopeptide. Nature 1981;289:593-4.

24 Beachey EH, Seyer JM, Dale JB, Simpson WA, Kang AH. Type-specific protective immunity evoked by synthetic peptide of Streptococcus pyogenes M protein. Nature 1981;292:457-9.

25 Rao KR, Vyas GN. Hepatitis B antigen activity in protein subunits produced by sonication. Nature New Biology 1973;241:240-1.

26 Zuckerman AJ, Howard CR. Prospects for hepatitis B vaccines. Nature 1973;246:445-7.

27 Zuckerman AJ. Hepatitis vaccine: a note of caution. Nature 1975;255: 104-5.

28 Zuckerman AJ. Developing synthetic vaccines. Nature 1982;295:98-9.

29 Hopp TP, Woods KR. Prediction of protein antigen acid sequences. Proc Natl Acad Sci USA 1981;78:3824-8.

30 Prince AM, Ikram $\mathrm{H}$, Hopp TP. Hepatitis B virus vaccine: identification of $\mathrm{HBsAg} / \mathrm{a}$ and $\mathrm{HBsAg} / \mathrm{d}$ but not $\mathrm{HBsAg} / \mathrm{y}$ subtype antigenic determinants on a synthetic immunogenic peptide. Proc Natl Acad Sci USA 1982;79:579-82.

31 Vyas GN. Molecular immunology of hepatitis B surface antigen (HBsAg). In: Maupas P, Guesry P, eds. Hepatitis B vaccine. Amsterdam: Elsevier/North Holland Biomedical Press, 1981:227. 36. (INSERM symposium No 18.)

32 Dreesman GR, Sanchez Y, Ionescu-Matiu I, et al. Production of antibody to hepatitis B surface antigen by single inoculation of uncoupled HBsAg peptides. Nature 1982;295:158-60.

33 Lerner RA, Green N, Alexander H, Liu F-T, Sutcliffe G, Shinnick TM. Chemically synthesized peptides predicted from the nucleotide sequence of the hepatitis $B$ virus genome elicit antibodies reactive with the native envelope protein of Dane particles. Proc Natl Acad Sci USA $1981 ; 78: 3403-7$.

34 Gerin JL, Alexander H, Shih J W-K, et al. Chemically synthesized peptides of hepatitis B surface antigen duplicate the $\mathrm{d} / \mathrm{y}$ specificities and induce subtype-specific antibodies in chimpanzees. Proc Natl Acad Sci USA 1983;80:2365-9.

35 Brown SE, Howard CR, Zuckerman AJ, Steward MW. Determination of the affinity of antibodies to hepatitis B surface antigen in human sera. $\mathcal{F}$ I mmunol Methods 1984;72:41-8.

36 Brown SE, Howard CR, Zuckerman AJ, Steward MW. Affinity of antibody responses in man to hepatitis B vaccine determined with synthetic peptides. Lancet 1984;ii:184-7. 